\title{
A Micromachined Nanopositioner With On-Chip Electrothermal Actuation and Sensing
}

\author{
Y. Zhu, Member, IEEE, A. Bazaei, S. O. R. Moheimani, Senior Member, IEEE, and \\ M. R. Yuce, Senior Member, IEEE
}

\begin{abstract}
This letter describes the design of a micromachined nanopositioner with thermal actuation and sensing capabilities in a single chip. The positioner has a dynamic range of $14.4 \mu \mathrm{m}$, and the sensor drift is $8.9 \mathrm{~nm}$ over $2000 \mathrm{~s}$ with a differential sensing scheme. The on-chip displacement sensing enables a feedback control capability. A proportional-integral feedback controller is designed and implemented digitally. The closed-loop step response results show a positioning resolution of $7.9 \mathrm{~nm}$ and a time constant of $1.6 \mathrm{~ms}$.
\end{abstract}

Index Terms-Feedback control, microelectromechanical systems (MEMS), nanopositioning, thermal actuation, thermal position sensing.

\section{INTRODUCTION}

$\mathbf{H}$ IGH-PRECISION nanopositioners have been used extensively in many applications such as scanning probe microscopy [1], atomic force microscopy [2], and emerging ultrahigh-density probe storage system [3], [4]. Closed-loop feedback control of the positioners is highly desirable if a high degree of displacement precision is required, and such a control system needs an accurate source of position information [5]. However, in microelectromechanical systems (MEMS) nanopositioners, the in-plane movements are often measured by laser reflectance microscopes [6], [7] or optical microscopes [8], making the footprint of the whole system fairly large. There are few exceptions in the literature, for example, an embedded on-chip capacitive displacement sensor was integrated in a thermally actuated positioner in [9]. Nevertheless, only open-loop results were obtained, and a complex fabrication process was required for electrical insulation between electrical heating and sensing circuits. Recently, a novel thermal sensing scheme was used in a probe-based storage device [10]. Microheaters were used to measure the motion of a MEMS microscanner with a resolution of less than $1 \mathrm{~nm}$. Compared to comb capacitive sensing, thermal sensing is more compact and can be easily integrated with actuators. In [11] and [12], off-chip electromagnetic coil actuators were adopted for scanner actuation, and

Manuscript received June 9, 2010; revised July 4, 2010; accepted July 5, 2010. Date of publication August 23, 2010; date of current version September 24, 2010. This work was supported by the Australian Research Council Discovery Grant-DP0774287. The review of this letter was arranged by Editor J. K. O. Sin.

The authors are with the School of Electrical Engineering and Computer Science, The University of Newcastle Australia, Callaghan, N.S.W. 2308, Australia (e-mail: yong.zhu@newcastle.edu.au; ali.bazaei@newcastle.edu.au; reza.moheimani@newcastle.edu.au; mehmet.yuce@newcastle.edu.au).

Color versions of one or more of the figures in this letter are available online at http://ieeexplore.ieee.org.

Digital Object Identifier 10.1109/LED.2010.2058841

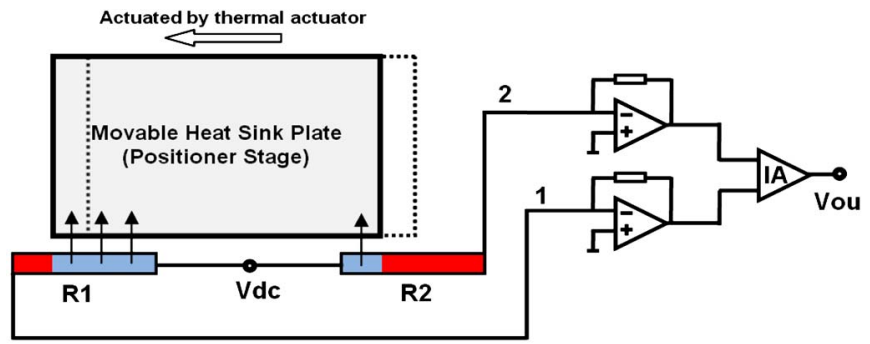

(a)

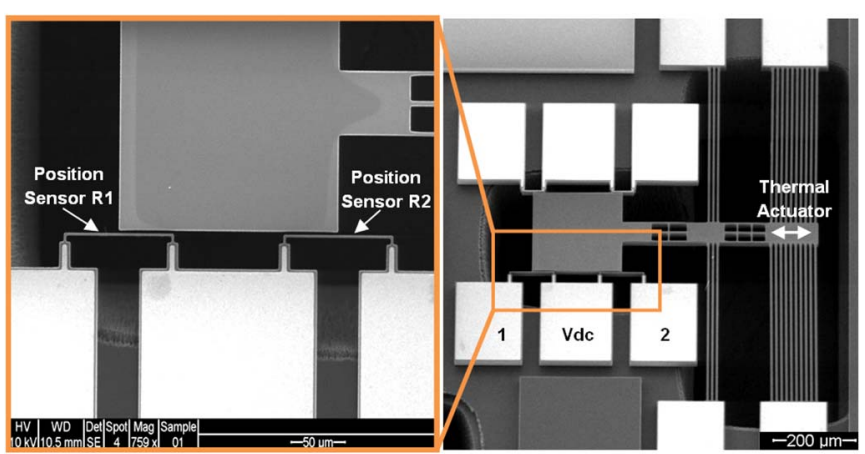

(b)

Fig. 1. (a) Schematic diagram of the thermal position sensor with a differential amplifier circuit. (b) SEM images of the micromachined nanopositioner.

a complex mass-balanced structure was designed for vibration resistance purposes.

In this letter, a novel thermal position sensor is integrated with a thermal actuator in the same MEMS chip without the need for inclusion of extra electrical insulation fabrication process [9] or assembling two chips as in [11]. Compared to other MEMS actuation mechanisms, the thermal actuators have advantages of low-voltage operation, large forces, and a high vibration resistance due to their stiff structures [13]. A MEMS device with integrated electrothermal actuation and sensing has been microfabricated in a bulk silicon process. Measurement results show that the positioner has a dynamic range of $14.4 \mu \mathrm{m}$ and an open-loop bandwidth of $101 \mathrm{~Hz}$. A model of the positioner is derived, and a proportional-integral (PI) feedback controller is implemented digitally in a dSPACE rapid prototyping system to investigate the closed-loop performance of the positioner. The closed-loop step response results show a positioning resolution of $7.9 \mathrm{~nm}$ and a time constant of $1.6 \mathrm{~ms}$.

\section{DESIGN}

The conceptual schematic view of the nanopositioner is shown in Fig. 1(a). The device is microfabricated from 
single-crystal silicon using a commercial bulk silicon micromachining technology-SOIMUMP in MEMSCAP [14]. This process has a $25-\mu \mathrm{m}$-thick silicon device layer and a minimum feature/gap of $2 \mu \mathrm{m}$. A scanning electron microscope (SEM) image of the whole device and a section of it are shown in Fig. 1(b). The position sensors are two beam-shaped resistive heaters made from doped silicon. Application of a fixed dc voltage across the heaters results in a current passing through them, thereby heating the beams. As a heat sink, a rectangle plate is placed beside the beam heaters with a $2-\mu \mathrm{m}$ air gap. The positioner stage is actuated by a thermal actuator, as shown in Fig. 1(b).

Before applying a voltage across the actuator, the positioner stage is at the initial rest position [dashed box in Fig. 1(a)], where the two edges of the sink plate are exactly aligned with the middle of the two thermal resistive sensors $R_{1}$ and $R_{2}$. The sensors are biased by a dc voltage source $V_{\mathrm{dc}}$, and the heat generated in the resistive heater is conducted through the air to the heat sink plate (positioner stage). As the plate is centered between the two thermal sensors, the heat fluxes out of the sensors are identical, thereby equaling the temperature and resistance of the sensors. After applying a voltage on the actuator beams, the positioner stage is displaced toward left, and the heat flux associated with the sensor on the left increases while that of the sensor on the right decreases, resulting in a decrease in the resistance of the left sensor $\left(R_{1}\right)$ and an increase in resistance of the right sensor $\left(R_{2}\right)$. Thus, the displacement information of the positioner stage can be detected by measuring the resistance difference between the two sensors. The differential changes of the resistance result in current variations in the beam resistors, and the currents are converted to an output voltage using transimpedance amplifiers and an instrumentation amplifier. To suppress the common-mode noise, the gains of these two transimpedance amplifiers must be well matched. Employing the differential topology allows the sensor output to be immune from undesirable drift effects due to changes in ambient temperature or aging effects.

\section{EXPERIMENTAL RESULTS AND DiscusSiON}

The nanopositioner was calibrated using a Polytec Planar Motion Analyzer. Digital image capture and analysis methods were used to determine the displacement of the positioner stage. The static actuation voltage versus displacement results are shown in Fig. 2(a). With an actuation voltage of $9 \mathrm{~V}$, the thermal actuator can achieve a maximum displacement of $14.4 \mu \mathrm{m}$. Meanwhile, at every actuation voltage, the instrumentation amplifier outputs were recorded for calibration of the position sensors, as shown in Fig. 2(b). The sensors were biased with $6 \mathrm{~V}$, and the instrumentation amplifier gain was set at $90.3 \mathrm{~V} / \mathrm{V}$. At this bias voltage, the sensors have a power consumption of $120 \mathrm{~mW}$ and a sensitivity of $0.27 \mathrm{mV} / \mathrm{nm}$. The dynamic characterization was conducted using an HP35670A spectrum analyzer. A voltage of $4.5-\mathrm{V}$ dc plus $1-\mathrm{V}$ ac was applied to the actuator, and swept sinusoidal measurements were obtained from $1 \mathrm{~Hz}$ to $51.2 \mathrm{kHz}$. The frequency response shown in Fig. 3 shows that the open-loop bandwidth of the positioner is $101 \mathrm{~Hz}$.
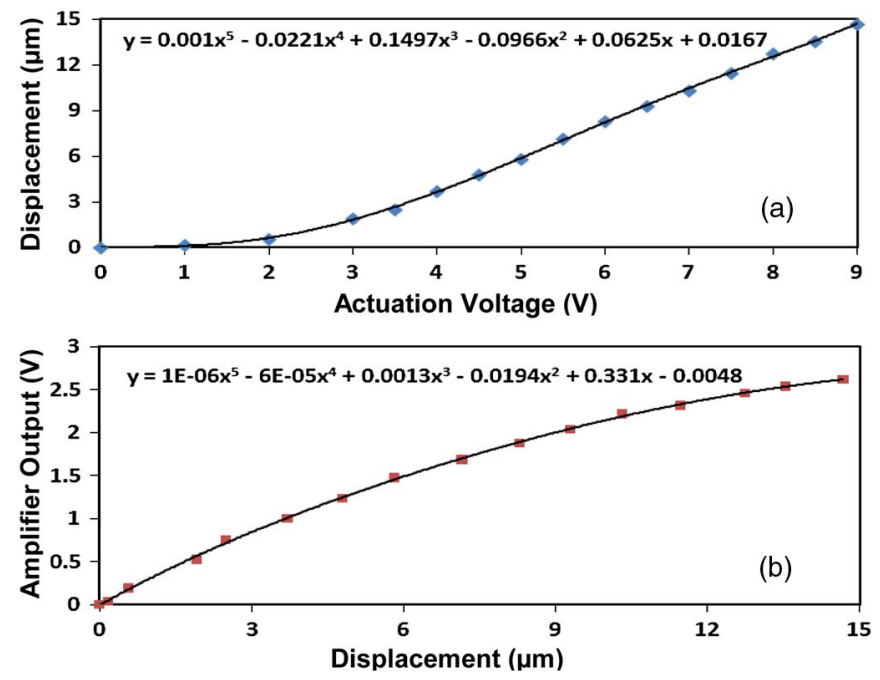

Fig. 2. Experimental calibration results. (a) Actuator displacement versus actuation voltage. (b) Instrumentation amplifier output versus actuator displacement.
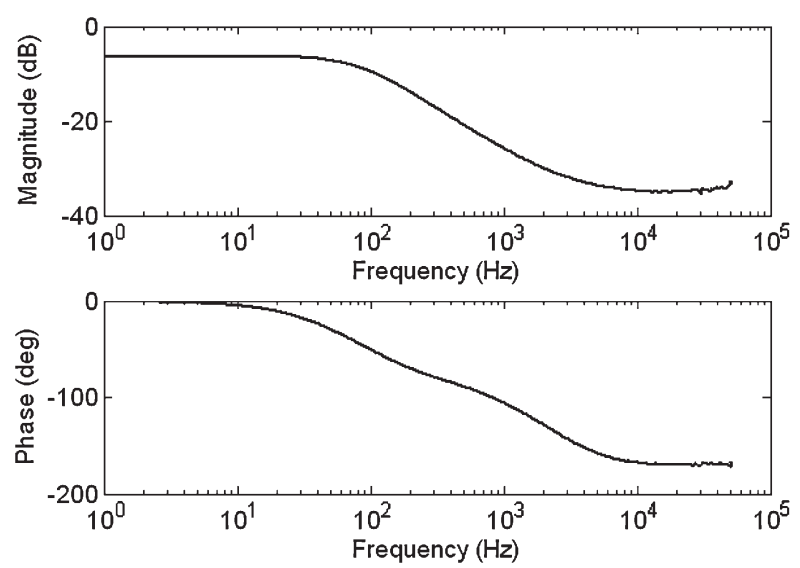

Fig. 3. MEMS nanopositioner experimental frequency response.

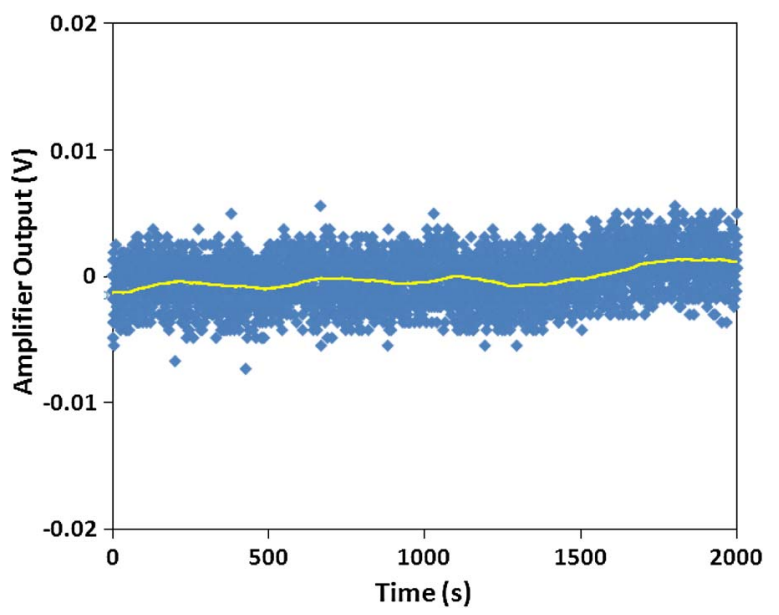

Fig. 4. Experimental results of sensor drift (open loop).

Fig. 4 shows the open-loop sensor drift when the positioner is at rest, which was measured at the output of the instrumentation amplifier over a period of $2000 \mathrm{~s}$ under normal laboratory conditions. The yellow/gray line is the moving average of the 


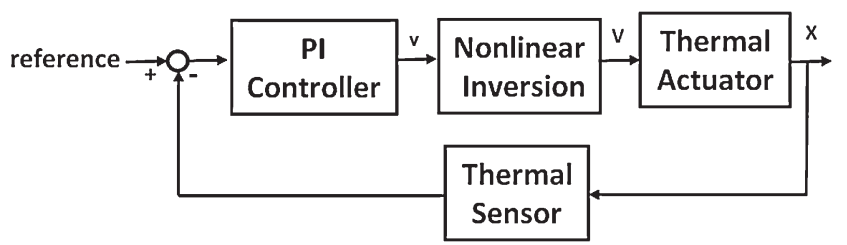

Fig. 5. Feedback loop block diagram including a PI controller.

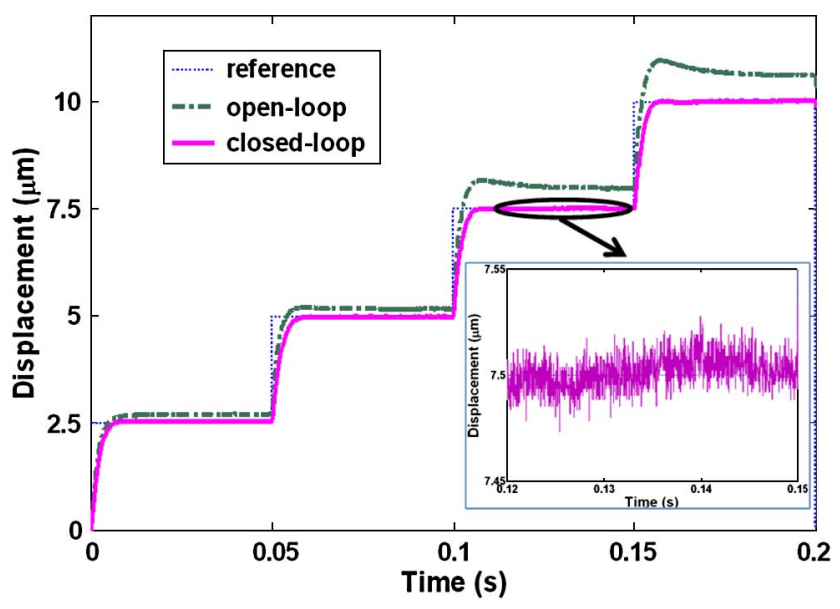

Fig. 6. Closed- and open-loop experimental results for 2.5, 5, 7.5, and $10 \mu \mathrm{m}$ seek operations.

measured data, which indicates the low-frequency drift. Owing to the differential sensing of the sensor pair, the open-loop amplifier output has a low drift of $2.4 \mathrm{mV}$ over $2000 \mathrm{~s}$, which corresponds to 8.9-nm displacement.

The PI closed-loop feedback control of the developed positioner was investigated to improve positioning accuracy and robustness of the system. The feedback loop block diagram including a PI controller is shown in Fig. 5. Due to the nonlinear nature of the thermal actuator, a nonlinear inversion block was added to the feedback loop to linearize the plant. Based on this control scheme, a controllable desired response of $2.5-\mu \mathrm{m}$ steps over a $10-\mu \mathrm{m}$ range was obtained with a positioning resolution of $7.9 \mathrm{~nm}$ and a time constant of $1.6 \mathrm{~ms}$, as shown in Fig. 6. As a comparison, a similar open-loop seek operation resulted in a maximum positioning error of $0.62 \mu \mathrm{m}$.

\section{CONCLUSION}

A novel micromachined silicon nanopositioner with on-chip thermal actuator and sensor has been presented with nanometer resolution and low sensor drift. The MEMS positioner was embedded in a feedback loop to realize a precise position control. Experimental results have shown that the positioner with PI controller achieved a high degree of positioning accuracy with very good robustness. A 2-D positioner is currently under investigation for imaging applications.

\section{REFERENCES}

[1] G. Binning and H. Rohrer, "The scanning tunneling microscope," Sci. Amer, vol. 253, pp. 50-56, 1986.

[2] G. Binning, C. Quate, and C. Gerber, "Atomic force microscope," Phys. Rev. Lett., vol. 56, no. 9, pp. 930-933, Mar. 1986.

[3] N. B. Hubbard, M. L. Culpepper, and L. L. Howell, "Actuators for micropositioners and nanopositioners," Appl. Mech. Rev., vol. 59, no. 6, pp. 324 334, Nov. 2006.

[4] A. Pantazi, M. A. Lantz, G. Cherubini, H. Pozidis, and E. Eleftheriou, "A servomechanism for a micro-electro-mechanical-system-based scanning-probe data storage device," Nanotechonology, vol. 15, no. 10, pp. S612-S621, Oct. 2004.

[5] S. Devasia, E. Eleftheriou, and S. O. R. Moheimani, "A survey of control issues in nanopositioning," IEEE Trans. Control Syst. Technol., vol. 15, no. 5, pp. 802-823, Sep. 2007.

[6] J. J. Gorman, Y-S. Kim, and N. G. Dagalakis, "Control of MEMS nanopositioners with nano-scale resolution," in Proc. IMECE, Chicago, IL, Nov. 5-10, 2006.

[7] R. Hichey, D. Sameoto, T. Hubbard, and M. Kujath, "Time and frequency response of two-arm micromachined thermal actuators," J. Micromech. Microeng., vol. 13, no. 1, pp. 40-46, 2003.

[8] Y. Sun, M. A. Greminger, D. P. Potasek, and B. J. Nelson, "A visually servoed MEMS manipulator," in Experimental Robotics VIII. Berlin, Germany: Springer-Verlag, 2003, pp. 255-264.

[9] L. L. Chu and Y. B. Gianchandani, "A micromachined 2D positioner with electrothermal actuation and sub-nanometer capacitive sensing," J. Micromech. Microeng., vol. 13, no. 2, pp. 279-285, Mar. 2003.

[10] M. A. Lantz, G. K. Binning, M. Despont, and U. Drechsler, "A micromechanical thermal displacement sensor with nanometre resolution," Nanotechnology, vol. 16, no. 8, pp. 1089-1094, 2005.

[11] A. Sebastian, A. Pantazi, S. O. R. Moheimani, H. Pozidis, and E. Eleftheriou, "Achieving subnanometer precision in a MEMS-based storage device during self-servo write process," IEEE Trans. Nanotechnol., vol. 7, no. 5, pp. 586-595, Sep. 2008.

[12] M. A. Lantz, H. E. Rothuizen, U. Drechsler, W. Haberle, and M. Despont, "A vibration resistant nanopositioner for mobile parallel-probe storage applications," J. Microelectromech. Syst., vol. 16, no. 1, pp. 130-139, Feb. 2007.

[13] V. Kaajakari, Practical MEMS. Las Vegas, NV: Small Gear Publishing, 2009.

[14] [Online]. Available: http://www.memscap.com/en_mumps.html 